\title{
Distinct Modulatory Effects of Satiety and Sibutramine on Brain Responses to Food Images in Humans: A Double Dissociation across Hypothalamus, Amygdala, and Ventral Striatum
}

\author{
Paul C. Fletcher, ${ }^{1}$ Antonella Napolitano, ${ }^{2}$ Andrew Skeggs, ${ }^{2}$ Sam R. Miller, ${ }^{2}$ Bruno Delafont, ${ }^{2}$ Victoria C. Cambridge, ${ }^{1}$ \\ Sanne de Wit, ${ }^{3}$ Pradeep J. Nathan, ${ }^{1,2}$ Allison Brooke, ${ }^{2}$ Stephen 0'Rahilly, ${ }^{4}$ I. Sadaf Farooqi, ${ }^{4}$ and Edward T. Bullmore ${ }^{1,2}$ \\ ${ }^{1}$ Department of Psychiatry, University of Cambridge, Cambridge CB2 0SZ, United Kingdom, ${ }^{2}$ GlaxoSmithKline Clinical Unit Cambridge, Addenbrooke's \\ Centre for Clinical Investigations, Addenbrooke's Hospital, Cambridge CB2 2QQ, United Kingdom, ${ }^{3}$ Amsterdam Center for the Study of Adaptive Control in \\ Brain and Behavior, Department of Developmental Psychology, University of Amsterdam, 1018 WB Amsterdam, The Netherlands, and ${ }^{4}$ University of \\ Cambridge Metabolic Research Laboratories, Institute of Metabolic Science, Addenbrooke's Hospital, Cambridge CB2 2QQ, United Kingdom
}

We used functional magnetic resonance imaging to explore brain responses to food images in overweight humans, examining independently the impact of a prescan meal ("satiety") and the anti-obesity drug sibutramine, a serotonin and noradrenaline reuptake inhibitor. We identified significantly different responses to these manipulations in amygdala, hypothalamus, and ventral striatum. Each region was specifically responsive to high-calorie compared to low-calorie food images. However, the ventral striatal response was attenuated by satiety (but unaffected by sibutramine), while the hypothalamic and amygdala responses were attenuated by drug but unaffected by satiety. Direct assessment of regional interactions confirmed the significance of this double dissociation. We explored the regional responses in greater detail by determining whether they were predictive of eating behavior and weight change. We observed that across the different regions, the individual-specific magnitude of drug- and satiety-induced modulation was associated with both variables: the sibutramine-induced modulation of the hypothalamic response was correlated with the drug's impact on both weight and subsequently measured ad libitum eating. The satiety-induced modulation of striatal response also correlated with subsequent ad libitum eating. These results suggest that hypothalamus and amygdala have roles in the control of food intake that are distinct from those of ventral striatum. Furthermore, they support a regionally specific effect on brain function through which sibutramine exerts its clinical effect.

\section{Introduction}

Characterization of pathways regulating energy homeostasis, largely using rodent models, has led to the identification of neural circuits modulating energy balance and nutrient partitioning (Morton et al., 2006). Functional magnetic resonance imaging (fMRI) is used increasingly to relate these insights to humans, offering the opportunity to explore factors such as weight, appetite, eating behaviors, and food type (Killgore and YurgelunTodd, 2005; Porubská et al., 2006; Rothemund et al., 2007; Führer et al., 2008; Coletta et al., 2009; McCaffery et al., 2009; Schienle et al., 2009). It has also been possible to link brain responses to levels

\footnotetext{
Received June 28, 2010; revised Aug. 18, 2010; accepted Aug. 27, 2010.

The study was sponsored by GlaxoSmithKline. P.C.F. was supported by the Wellcome Trust and by the Bernard Wolfe Health Neuroscience Fund. S.O.R. and I.S.F. were supported by the Wellcome Trust, Medical Research Council (MRC) Centre for Obesity and Related Disorders, and National Institute for Health Research Cambridge Biomedical Research Centre. The work was also carried out in the MRC- and Wellcome Trust-funded Behavioural and Clinical Neurosciences Institute.

Correspondence should be addressed to either of the following: I. Sadaf Farooqi, University of Cambridge Metabolic Research Laboratories, Institute of Metabolic Science, Addenbrooke's Hospital, Cambridge CB2 20Q, UK, Email: isf20@cam.ac.uk; or Paul C. Fletcher, Department of Psychiatry, University of Cambridge, Herchel Smith Building for Brain and Mind Sciences, Cambridge CB2 0SZ, UK, E-mail: pcf22@cam.ac.uk.

DOI:10.1523/JNEUROSCI.3323-10.2010

Copyright $\odot 2010$ the authors $\quad 0270-6474 / 10 / 3014346-10 \$ 15.00 / 0$
}

of peripheral hormones (Batterham et al., 2007; Farooqi et al., 2007; Malik et al., 2008), genes (Stice et al., 2008), gender (Smeets et al., 2006; Uher et al., 2006; Killgore and Yurgelun-Todd, 2010), personality (Beaver et al., 2006), and mood (Killgore and Yurgelun-Todd, 2007).

In addition, advances in pharmacological fMRI approaches offer new ways of exploring neural contributions to eating behavior as well as elucidating the mechanisms of action of current and potential anti-obesity compounds. In this study, we determined the impact of two factors on brain responses to high-calorie foods: satiety and the centrally acting anti-obesity drug, sibutramine. The experimental design is summarized in Figure 1.

The first factor, satiety, has been shown to have an impact on brain responses to food-related stimuli. While there has been some variability, separate studies have noted that the fasted state is associated with greater activation in amygdala (LaBar et al., 2001; Holsen et al., 2005; Smeets et al., 2006; Führer et al., 2008; Goldstone et al., 2009; Piech et al., 2009), insula (Pelchat et al., 2004; Holsen et al., 2006; Porubská et al., 2006; Uher et al., 2006; Cornier et al., 2009; Goldstone et al., 2009), striatal regions (Pelchat et al., 2004; Porubská et al., 2006; Smeets et 


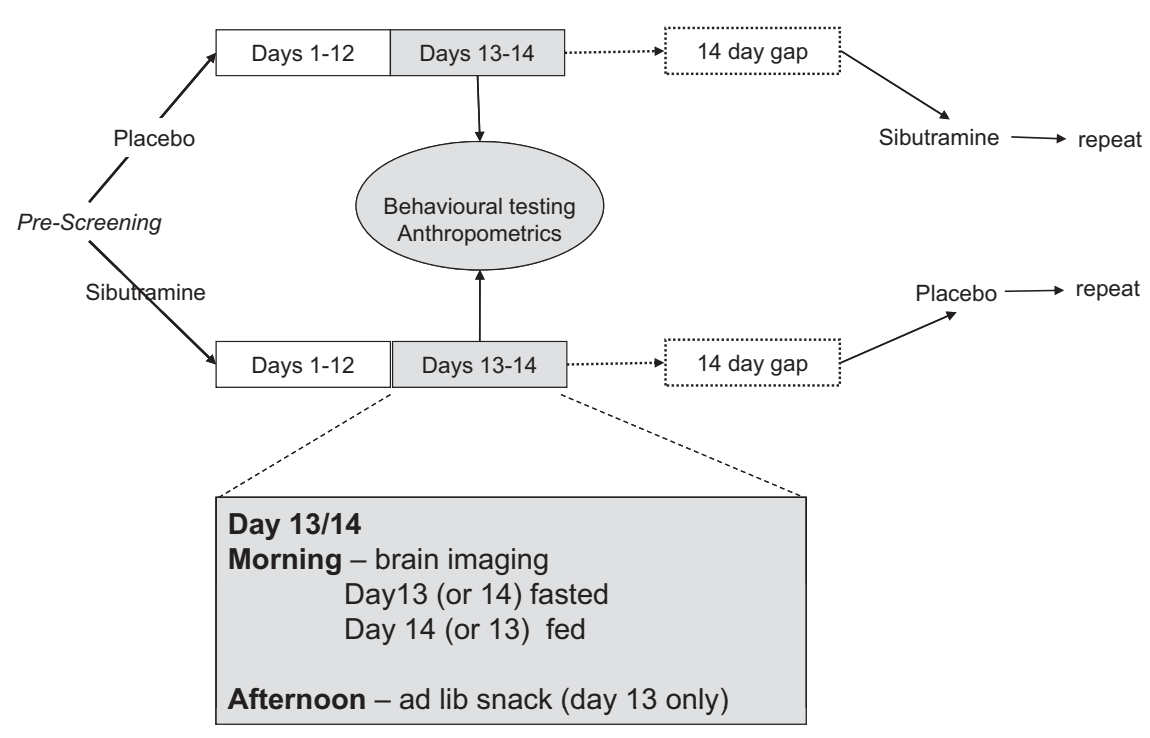

Figure 1. Schematic of study design.

http://www.ema.europa.eu/pdfs/human/referral/ sibutramine/3940810en.pdf), and was not recommended for treatment of patients with pre-existing heart disease. All the patients participating in the current study had normal cardiovascular function (established by medical history, physical examination, and repeated ECG) and regular clinical safety reviews, and were prescribed sibutramine within the existing therapeutic guidelines.

Note that key observations were made while participants were resident in the research unit. Critically, this enabled us to ensure full experimental control of fasted/fed state before each imaging session.

\section{Experimental paradigm}

In a double-blind, placebo-controlled, crossover design (see Fig. 1), participants were randomized immediately after baseline assessment to receive oral sibutramine $(15 \mathrm{mg} / \mathrm{d}$ in a single morning dose) and oral placebo, each for a period of $14 \mathrm{~d}$, in counterbalanced order. Close to the end of each treatment pe-

al., 2006; Goldstone et al., 2009), and hypothalamus (Smeets et al., 2006; Cornier et al., 2007, 2009).

The second factor involved a 2 week period of treatment with sibutramine, a centrally acting drug that was, until recently, licensed for weight reduction in obesity. Long-term trials have shown that patients treated with sibutramine achieve body weight reduction of $\sim 4-5 \%$ greater than those treated with placebo (Hansen et al., 2001). Sibutramine acts principally by blocking synaptic reuptake of serotonin and noradrenaline (Nisoli and Carruba, 2000; Lean, 2001), but how these effects on synaptic transmission lead ultimately to therapeutic changes in eating behavior is unknown.

In brief, using a randomized, placebo-controlled, withinsubjects, counterbalanced design, we examined the impact on neural responses of natural (eating-induced) satiety, as well as the pharmacologically induced reduction in desire for food. We related neural effects to relevant anthropometric (body weight and fat mass) and behavioral (ad libitum food intake) variables recorded outside the scanner. In this way, we sought to establish, more specifically, the different contributions of brain regions to eating behaviors and to explore the mechanism of action of sibutramine.

\section{Materials and Methods Subjects}

Participants were recruited by local advertisement. Prospective inclusion criteria were age ( $18-45$ years), body mass index (BMI) of between 25 and 30 , and lack of medical conditions (except asthma) or drug or alcohol dependence. Twenty-eight overweight, healthy volunteers [mean age 34.3 ( \pm 7.3 ) years; 22 male] were recruited. Technical problems limit the complete fMRI datasets presented here to 24. Patient characteristics of these 24 participants at baseline are summarized in Table 1 (characteristics of the complete set of 28 participants are summarized in supplemental Table 1, available at www.jneurosci.org as supplemental material). Blood glucose and thyroid-stimulating hormone levels were checked to be normal at baseline. The study was approved by a local regional ethics committee in Cambridge (REC ref 08/H0308/11) and was conducted at GlaxoSmithKline's Clinical Unit in Cambridge, Addenbrooke's Hospital, Cambridge, UK, in accordance with the principles of the Declaration of Helsinki. All participants provided written, informed consent after the nature and possible consequences of the studies were explained.

The experimental treatment, sibutramine, is now known to be associated with increased cardiovascular risk following prolonged treatment trials (see riod (day 12), participants were admitted for $2 \mathrm{~d}$ to the clinical unit, where they underwent anthropometric, behavioral, and functional MRI assessments as detailed below. Between treatments, there was a 2 week washout period.

\section{Assessments}

\section{Anthropometric}

Body weight and BMI were measured conventionally. Body composition was measured using a low-field magnetic resonance instrument, QMR Echo-MRI, providing measure of total body fat mass, lean mass, and total body water. Ad libitum food intake was measured by allowing participants to eat as much popcorn as they wished while watching an entertaining television program for $20 \mathrm{~min}$. The amount consumed in grams was subsequently correlated with key imaging observations (as described below). Note that one of the 24 scanned participants did not like popcorn, and his data were not used in subsequent correlation analyses.

\section{fMRI}

Participants were scanned twice using fMRI (on the morning of both assessment days at $\sim 8: 30$ A.M.) at the end of each treatment period. For one of the scanning sessions, participants had fasted from 10 P.M. the previous evening; for the other, they had eaten a standard breakfast $\sim 30$ min before arriving at the scanner. The order of fasted and fed scanning sessions was counterbalanced across participants.

\section{Imaging task and technical details}

In each 30 min session, participants were scanned while watching a series of images of high-calorie food items (e.g., chocolate), low-calorie foods (e.g., broccoli), or nonfood items [everyday objects (watches, jewelry, clothing) and scenes], matched across categories for color and other visual properties. Four sets of 75 images ( 25 high-calorie food, 25 lowcalorie food, 25 nonfood) were selected with a different set being used on each of the four scanning sessions (the order of sets used was counterbalanced across participants for the four sessions). Images from each category were presented in counterbalanced order in blocks of five (each image appearing for $5 \mathrm{~s}$ ) interspersed with periods of fixation, each block lasting $25 \mathrm{~s}$. Participants were instructed to think about how much they liked each image and push a button in response to each.

Participants also performed a separate Pavlovian and instrumental learning task, which will be analyzed and reported elsewhere. There was no overlap of stimuli across the two tasks. The learning task was performed first in each scanning session.

\section{fMRI data acquisition and analysis}

We used a Siemens Trio scanner operating at 3 tesla with a $192 \mathrm{~mm}$ field of view in the Wolfson Brain Imaging Centre, Cambridge. A total of 505 


\section{Table 1. Scanned sample characteristics}

\begin{tabular}{lc}
\hline & Subjects undergoing fMRI (24) \\
\hline Age (years) & \\
Mean (SD) & $34.9(6.89)$ \\
Range & $22-44$ \\
Sex & \\
Male & $19(79 \%)$ \\
Female & $5(21 \%)$ \\
Ethnicity & \\
Black & $0(0 \%)$ \\
Caucasian & $22(92 \%)$ \\
Other & $2(8 \%)$ \\
Weight (kg) & \\
Mean (SD) & $85.2(7.54)$ \\
Range & $71.3-102.5$ \\
BMI (kg/m $\left.{ }^{2}\right)$ & \\
Mean (SD) & $27.06(1.40)$ \\
Range & $25.3-30.6$ \\
Fat mass (kg) & \\
Mean (SD) & $21.1(6.63)$ \\
Range & $9.7-39.4$ \\
Barratt Impulsiveness Scale & \\
Mean (SD) & $62.1(7.58)$ \\
Range & $47-79$ \\
\hline
\end{tabular}

gradient echo $\mathrm{T} 2{ }^{\star}$-weighted echo-planar images depicting blood oxygenation level-dependent contrast were acquired for each participant. The first six images were treated as "dummy" scans and discarded to avoid T1 equilibration effects. Images were positioned at $30^{\circ}$ to the anterior commissure-posterior commissure plane and comprised 32 slices, each of $3 \mathrm{~mm}$ with a $0.5 \mathrm{~mm}$ interslice gap and $64 \times 64$ data matrix. A repetition time of $2000 \mathrm{~ms}$ was used with an echo time of $30 \mathrm{~ms}$ and $90^{\circ}$ flip angle.

Data were analyzed using statistical parametric mapping in the SPM5 program (www.fil.ion.ucl.ac.uk). Images were realigned then spatially normalized to a standard template and spatially smoothed with a Gaussian kernel ( $8 \mathrm{~mm}$ full width at half-maximum). The time series in each session were high-pass filtered (with cutoff frequency $1 / 120 \mathrm{~Hz}$ ) and serial autocorrelations were estimated using an AR(1) model. The blocked conditions were modeled using a canonical hemodynamic response function convolved with a boxcar function placed at the onset of each block. For each set of scans, we modeled the three conditions (highcalorie food, low-calorie food, nonfood), which were specified as covariates in a general linear model, and an activation parameter was estimated at each voxel for each stimulus type. The parameter estimate, derived from the mean least-squares fit of the model to the data, reflects the strength of the covariance between the data and the canonical response function for a given stimulus condition. The responses to each condition were compared to the fixation baseline, and these contrasts were taken forward to a group analysis treating intersubject variability as a random effect. At the group level, for the condition effects, we used an ANOVA model to explore the effects of stimulus type, satiety, and treatment. As detailed below, we explored the main effect of stimulus type (both all food vs nonfood images and high-calorie vs low-calorie images) before establishing the modulatory effects of drug and satiety on stimulusrelated neural responses. The drug, satiety, and condition effects were specified as non-independent (repeated measures) having unequal variance. The violation of sphericity in the ensuing covariance components are estimated in SPM, using Restricted Maximum Likelihood with corresponding adjustment of the statistics and degrees of freedom.

In a further post hoc analysis motivated by our observations of hypothalamic responses (see below), we aimed to reliably localize individualspecific high-calorie food-related activations, by reanalyzing images with a smaller Gaussian smoothing kernel (4 mm full width at halfmaximum) and mapping activations onto each individual's own spatially normalized structural MRI. These data are presented in supplemental Figure 1 (available at www.jneurosci.org as supplemental material).
Defining regions of interest

In analyzing these data, we used existing knowledge of the functional neuroanatomy of food reward processing and eating behavior (Porubská et al., 2006; Farooqi et al., 2007; Führer et al., 2008) to focus attention on the following brain regions: insula, ventral and dorsal striatum, hypothalamus, amygdala, and midbrain. This approach reduced the number of statistical tests, compared to that entailed in a theoretically uninformed analysis of the whole brain. A standard procedure for correction of false-positive error rates was used to adjust significance levels for multiple comparisons.

Regions of interest for the analyses relating to the main effects of image type (all food vs nonfood and high-calorie vs low-calorie images) were anatomically defined using the Pickatlas tool (Maldjian et al., 2003) implemented in SPM5 and consisted of the following: nucleus accumbens, caudate nucleus, putamen, globus pallidus, midbrain, hypothalamus, and amygdala. The midbrain region was defined within a sphere with radius $=12 \mathrm{~mm}$ centered at $x, y, z=0,-15,-9 \mathrm{~mm}$, as in previous work from our group (Murray et al., 2008). A familywise error correction for the entire set of regions of interest was used. Subsequently, regional activations for the main effects (high-calorie vs low-calorie food images) were used for further region of interest analyses. We carried this out using the initial analysis (high-calorie vs low-calorie images for all drug and satiety conditions) to identify the key foci showing a calorie-specific response and then testing for the specific interactions (task by satiety, task by drug, task by satiety by drug). These interactions are orthogonal to the main effects. For each region, therefore, we identified left- and right-sided foci and tested interactions within spheres $(d=10 \mathrm{~mm})$ centered around the foci, using a standard Bonferroni correction for the overall number of tests (six). (An exception was the insula, which is a large structure and contained several foci of calorie-specific activation. We therefore used an anatomical mask of the entire insula bilaterally to test for interactions in this region, correcting for multiple comparisons appropriately.)

\section{Analyses}

Brain responses to picture stimuli regardless of satiety or sibutramine treatment

Two contrasts are reported reflecting overall brain responses within the regions of interest to images viewed: the primary analysis identified the specific impact of calorific value, i.e., the main effect of viewing highcalorie compared to low-calorie images; a second analysis assessed the main effect of all food pictures (high and low calorie) compared to nonfood items. The latter analysis was performed for completeness and is reported in supplemental Table 1 (available at www.jneurosci.org as supplemental material). The purpose of the first analysis was to identify key regions within the system that were specifically responsive to the highcalorie (compared to low-calorie) food images. This was the basis for further identification of the drug and fasting effects. That is, in all subsequent analyses, we focus specifically on the regions showing significantly greater responses to high-calorie than to low-calorie images. We ensured this by using this analysis to identify a subset of regional activations with all subsequent tests being performed on spheres drawn around these regions (diameter of sphere of interest $=10 \mathrm{~mm}$ ). These analyses were subject to small volume correction on this basis.

In short, our aim in this set of complementary analyses was to identify calorie-specific brain activations within a set of regions suggested by prior imaging work and then to subject the subset of regions showing such calorie-specific activation in the current study to a set of orthogonal analyses identifying the main effects of sibutramine and satiety as well as the interaction between these two factors.

Modulatory effects of satiety on calorie-responsive brain activation Regional activation patterns. We were primarily interested in which of the regions specifically activated in response to high-calorie images showed a modulation of activity depending on whether they were fasted or fed but regardless of drug treatment. This comparison explored, in essence, the main effect of satiety on calorie-specific activity.

Behavioral relevance of brain activation. Regions showing the fasting/ stimulus interaction were then assessed further by extracting parameter 
Table 2. Effects of sibutramine

\begin{tabular}{|c|c|c|c|c|c|c|c|c|c|}
\hline & \multicolumn{2}{|c|}{ Sibutramine } & \multicolumn{2}{|l|}{ Placebo } & \multirow[b]{2}{*}{ Difference } & \multirow[b]{2}{*}{$95 \% \mathrm{Cl}$} & \multirow[b]{2}{*}{$t$} & \multirow[b]{2}{*}{$\mathrm{df}$} & \multirow[b]{2}{*}{$p$ value } \\
\hline & Mean & SD & Mean & SD & & & & & \\
\hline Change in fat mass $(\mathrm{kg})$ & -0.76 & 0.55 & 0.04 & 0.62 & -0.79 & $(-1.14,-0.44)$ & 4.6 & 41 & $<0.0001$ \\
\hline Change in weight $(\mathrm{kg})$ & -1.85 & 1.19 & -0.31 & 1.07 & -1.56 & $(-2.27,-0.86)$ & 4.5 & 39 & $<0.0001$ \\
\hline Eating rate (g/min) & 27.5 & 8.9 & 29.2 & 10.4 & -2.6 & $(-6.5,1.3)$ & 1.4 & 18.5 & 0.1742 \\
\hline HCFQ total score & 18.0 & 2.6 & 20.8 & 2.7 & -2.9 & $(-4.2,-1.6)$ & 4.7 & 21.6 & 0.0001 \\
\hline Ad libitum eating (g) & 53.3 & 43.8 & 71.7 & 44.7 & -15.7 & $(-30.8,-0.5)$ & 2.2 & 20.9 & 0.0430 \\
\hline
\end{tabular}

estimates from foci of maximal effect and determining whether there was a significant correlation, across individuals, with subsequent amount of food consumed in the ad libitum "snack" task described above. The purpose of this analysis was to assess whether the stimulus-dependent activations showing sensitivity to fasted/fed state were predictive of behavior when given access to real food.

\section{Modulatory effects of sibutramine on calorie-responsive} brain activation

Regional activation patterns. Here, we determined whether regions specifically activated in response to high-calorie images showed a modulation of activity depending on whether they had received sibutramine or placebo during the previous 2 weeks, regardless of whether they were fasted or fed during the scanning session. This comparison explored, in essence, the main effect of sibutramine on calorie-specific activity.

Behavioral relevance of brain activation. Regions showing the drug modulation were then assessed further by extracting parameter estimates from maximal foci and determining whether there was a significant correlation, across individuals, with the drug's impact on the amount of food consumed in the ad libitum "snack" task described above. Here, the purpose of the analysis was to assess whether the drug's effect on brain responses was related to its effects on behavior. Further correlations were also performed with key outcome measures relating to sibutramine's clinical effects [weight reduction, loss of fat mass, changes in Hunger, Craving, Fullness Questionnaire (HCFQ) score].

\section{Post hoc analyses}

We performed two post hoc analyses to inform our understanding of the drug effects more clearly.

Post hoc analysis 1: are sibutramine-induced changes dissociable from nonspecific effects of weight loss? Since any differences in brain response could be directly attributable to the drug but might also be a secondary effect of the anticipated weight loss, we performed a post hoc analysis assessing those individuals who received sibutramine before placebo. In these individuals it was anticipated that weight after the initial 2 weeks of sibutramine treatment would be comparable to that measured 4 weeks later when they had finished the placebo arm. That is, for this subgroup, any differences in brain response would be more directly attributable to sibutramine and could not be accounted for merely by weight change.

Post hoc analysis 2: are hypothalamic responses before treatment predictive of the clinical response to sibutramine? In a post hoc analysis, we explored correlations between brain response under placebo treatment and subsequent weight/fat loss on sibutramine in those individuals who had been randomized to receive placebo first. That is, we addressed the question of whether brain response before receiving sibutramine (i.e., at baseline response) would predict the efficacy of sibutramine when they later received it. This analysis sought to establish whether there is an individual variability that might predict, rather than merely reflect, the efficacy of subsequent sibutramine treatment.

Responses to high-calorie (compared to low-calorie) images differing as a consequence of both drug treatment and fasted/fed state

Finally, we explored the two-way interaction (fasted/fed state by drug/ placebo state) to identify regions in which the stimulus related activation showed a modulation by fasted state that differed across sibutramine and placebo states. This would give clues about whether sibutramine was having an effect on brain activity that was particularly determined by the subjects' current state of satiety.

\section{Results}

Sample characteristics are summarized in Table 1.

\section{Body weight/composition and eating behavior}

These effects are summarized in Table 2. Body weight was reduced to a greater extent following sibutramine, and this difference was highly significant (see Table 2). There was also a significantly greater reduction of fat mass following sibutramine (see Table 2), but no significant treatment difference in total body water $(p=0.18)$. Although reduction of body water has been considered an important component of weight loss in the short-term treatment of obesity, these data indicate that the most consistent contribution to the weight reduction following sibutramine is attributable to reduced fat mass. Blood pressures measured following each 2 week treatment period did not show a significant effect of sibutramine [mean systolic pressures: placebo mean $(\mathrm{SD})=113$ (11) $\mathrm{mmHg}$; sibutramine $=114$ (10) mmHg; mean diastolic pressures: placebo $=66(8) \mathrm{mmHg}$; sibutramine $=64(6) \mathrm{mmHg}]$. We are confident therefore that changed blood pressure did not have an impact on brain response measurements reported below.

Consistent with its effects on body weight, sibutramine was associated with significantly greater reductions in self-reported intensity of hunger and craving, and increases in subjective reports of satiety, measured using the HCFQ $(t=4.04 ; \mathrm{df}=32.2$; $p=0.0001)$. Sibutramine was also associated with significantly reduced ad libitum food intake $(t=2.2 ; \mathrm{df}=20.9 ; p=0.043)$.

\section{Brain responses to picture stimuli regardless of satiety or sibutramine treatment}

This initial analysis was performed to determine the brain regions responding to high-compared to low-calorie food images, regardless of drug or fasted state. As described in the methods section, we confined this analysis to a set of regions shown across a broad set of previous studies to be sensitive to food-related stimuli: orbitofrontal cortex, insula, midbrain, hypothalamus, amygdala, and ventral striatum. As expected, there was significantly greater activation in response to high-calorie food than in response to low-calorie food images in a number of predicted regions, notably insula, striatum, midbrain, hypothalamus, and amygdala. The results are summarized in Figure 2 and Table 3. Similar activations were found when comparing responses to all food images to responses to nonfood images (see supplemental Table 1, available at www.jneurosci.org as supplemental material).

Regions showing significantly greater activation for highcalorie than for low-calorie images (henceforth referred to as a "calorie-specific" response) provided the focus for subsequent analysis of satiety-dependent and drug-dependent modulations as described above. 
Modulatory effects of satiety on calorieresponsive brain activation

We observed a modulation of ventral striatal responses [nucleus accumbens/head of caudate nucleus; $x, y, z=8 \mathrm{~mm}, 6 \mathrm{~mm}$, $4 \mathrm{~mm} ; z=3$; small volume familywise error corrected for the volume of interest and Bonferroni corrected for the number of regions tested $(p=0.048)]$ according to whether participants viewed highcalorie images (compared to low-calorie images) in the fasted or fed state. As shown in Figures 2 and 3, the effect of satiety was to attenuate the ventral striatal response to high-calorie food images. The magnitude of satiety-related difference in ventral striatal activation was correlated with subsequent behavior when given access to real food. Significant correlations were found between the magnitude of the eating-related attenuation of caloriespecific striatal activation and the amount of popcorn consumed during that visit (Spearman's $\rho=0.42 ; p=0.025$, onetailed). See Figure 3.

\section{Modulatory effects of sibutramine on calorie-responsive brain activation}

We next determined the location of sibutramine's effects on brain responses. A significant impact on brain response to high (compared to low)-calorie food images was identified in two regions: right hypothalamus $[x, y, z=4 \mathrm{~mm},-2 \mathrm{~mm}$, $-6 \mathrm{~mm} ; Z=3.6$; small volume familywise error corrected for the volume of interest and Bonferroni corrected for the number of regions tested $(p=0.014)]$ and right amygdala $[x, y, z=22 \mathrm{~mm}, 1 \mathrm{~mm},-17$ $\mathrm{mm} ; Z=3.2$; small volume familywise error corrected for the volume of interest and Bonferroni corrected for the number of regions tested $(p=0.024)]$. See Figures 2 and 4 . Bearing in mind the small size of the hypothalamus, and the potential error in localizing it automatically in computationally normalized images, we corroborated this key result with supplementary individualized analyses with reduced spatial smoothing to optimize precision of localization. We superimposed each participant's functional response to high-calorie foods onto their own structural MRI scan (note that structural scan data of sufficient quality were unavailable for one individual). This confirmed the localization of activity to the hypothalamus in the majority of individuals (see supplemental Fig. 1, available at www. jneurosci.org as supplemental material). Note that while in some individuals the focus of maximal effect was on the right, in others it was on the left (the greatest groupwise effect was right-sided).

The modulatory effects of sibutramine on brain activation in the hypothalamus were significantly correlated with its effects
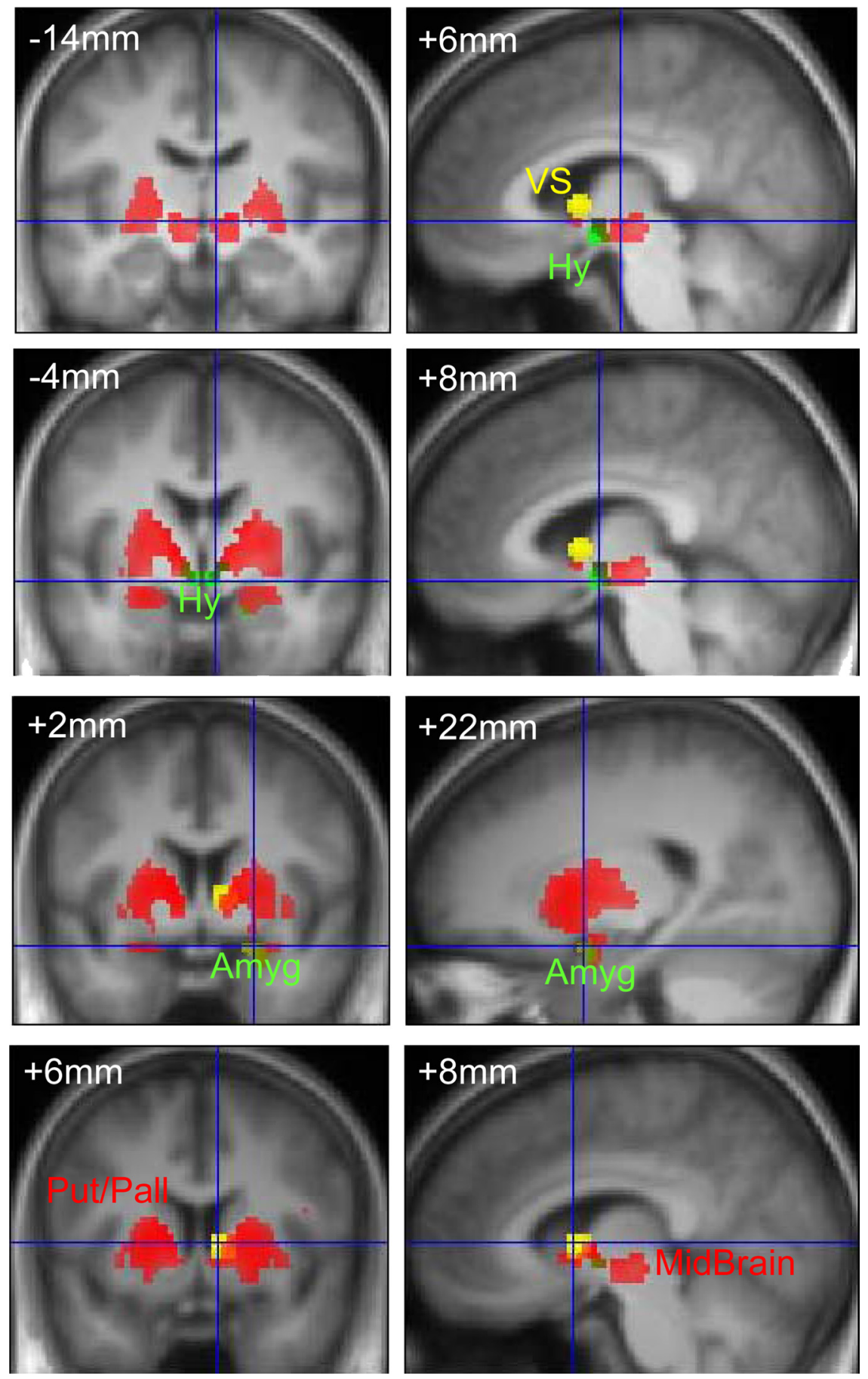

Figure 2. Brain activations in response to high-calorie food images, and modulations by sibutramine and satiety. Coronal (at $y=-14 \mathrm{~mm},-4 \mathrm{~mm},+2 \mathrm{~mm}$, and $+6 \mathrm{~mm}$ relative to the anterior commissure) and sagittal (at $x=+6 \mathrm{~mm},+8 \mathrm{~mm},+22$ $\mathrm{mm}$, and $+8 \mathrm{~mm}$ to the right of the midline) sections from the group average structural image are shown. Superimposed on these in red are significant activations from the comparison between high- and low-calorie food images (all surviving $p<0.05$, familywise error correction for multiple comparisons). Key regions showing this effect were insula, striatum [including putamen and globus pallidus (Put/Pall)], midbrain, amygdala, and hypothalamus. Superimposed in yellow is the region of ventral striatum (VS) showing a significantly greater response in the fasted state. In green are those regions - hypothalamus (Hy) and amygdala (Amyg) —-that showed a stimulus-type-by-drug interaction, i.e., significant reduction in high-calorie stimulus-related activation following treatment with sibutramine (compared to placebo). The interactions threshold is $p<0.01$, uncorrected, for display. on body weight (Spearman's $\rho=-0.51$, two-tailed $p=0.02$ ), and on ad libitum food intake (Spearman's $\rho=-0.58$, twotailed $p=0.004$ ); see Figure 2. Thus, individuals who experienced the greatest reduction in body weight and ad libitum food intake following sibutramine treatment also tended to 
Table 3. Greater brain responses to high-calorie than to low-calorie images (across all fasted/fed and drug/placebo conditions)

\begin{tabular}{lll}
\hline Region & Coordinates $(x, y, z)$ & Zscore \\
\hline $\begin{array}{l}\text { Ventral pallidum/putamen } \\
\quad \text { Left }\end{array}$ & $-12,2,4$ & 7.3 \\
& $-24,-18,4$ & 6.1 \\
$\quad$ Right & $12,2,2$ & 7 \\
Hypothalamus & $-10,-2,-4$ & 6.2 \\
$\quad$ Left & $8,-4,-3$ & 6 \\
$\quad$ Right & $10,4,2$ & \\
Ventral striatum & $-10,2,0$ & 7 \\
$\quad$ Right & & 6.9 \\
Left & $6,-14,-3$ & \\
Midbrain (substantia nigra/red nucleus) & $-8,-20,-10$ & 5.5 \\
$\quad$ Right & $-26,-3,-15$ & 5.3 \\
$\quad$ Left & $-18,-12,-9$ & 5.9 \\
Amygdala & $22,1,-17$ & 4.1 \\
$\quad$ Left & $20,-12,-10$ & 5.7 \\
Right & $32,11,-6$ & 3.6 \\
Insula & $42,17,-1$ & 5 \\
Right & $36,23,1$ & 5 \\
& $-42,17,-3$ & 4.5 \\
Left & $-32,10,02$ & 4.8 \\
& & 4.3 \\
\hline
\end{tabular}

All regions survived small volume correction for multiple comparisons.

show the greatest drug-related reduction in hypothalamic activation. However, sibutramine's effect on calorie-responsive brain activation in the hypothalamus was not significantly correlated with its effects on fat mass (Spearman's $\rho=-0.25$, $p=0.27$ ). There was also no evidence of significant correlation between sibutramine's effects on calorie-responsive activation in the amygdala and its effects on body weight, fat mass, or ad libitum eating behavior.

\section{Post hoc analysis 1: are the effects of sibutramine on brain activation dissociable from the effects of weight loss?}

Since sibutramine treatment was associated with significant weight reduction, and since weight reduction per se has been shown to modulate brain activation by food-related stimuli (Rosenbaum et al., 2008), one possible interpretation of sibutramine's effects on brain function is that they indirectly represent the pharmacologically nonspecific effects of weight reduction. To address this potential confound, we focused on the subgroup of participants who had been randomized to receive treatment with sibutramine before treatment with placebo, extracting parameter estimates from the hypothalamic and amygdala foci identified by the comparison above. We found that weight reduction following sibutramine was maintained following placebo, i.e., the subjects did not have significantly different body weight at the time of the fMRI scans conducted at the end of each treatment period. Yet the effect of sibutramine on hypothalamic $\left(F_{(1,10)}=10.4 ; p=\right.$ 0.005 , one-tailed) and amygdala $\left(F_{(1,10)}=6.3 ; p=0.02\right.$, onetailed) activation remained significant. This result suggests that sibutramine's attenuation of hypothalamic and amygdala activation by high-calorie food images is not simply attributable to the confounding effects of reduced body weight but more likely represents a pharmacologically specific effect of the drug.
Post hoc analysis 2: are hypothalamic responses before treatment predictive of the clinical response to sibutramine? Here, we determined whether brain responses measured before receiving sibutramine would predict its efficacy when later administered. Thus, confining an analysis to the subgroup of individuals who received placebo during the initial 2 week period (data available on 12 participants), we observed that the hypothalamic response to high-calorie foods following placebo was indeed predictive of later weight loss when sibutramine was started $(n=11$; Spearman's $\rho=-0.81, p=0.003)$. We should add that this analysis was performed as a preliminary test of whether brain activation in response to specific food stimuli might predict the efficacy of sibutramine before it had ever been taken. However, as the analysis is not statistically independent of the analysis performed to assess the correlation between weight loss and sibutramine differences relative to placebo, we treat this finding with due caution.

Responses to high-calorie (compared to low-calorie) images differing as a consequence of both drug treatment and fasted/fed state

None of the regions showing a calorie-specific response showed a significant interaction between drug and fasted/fed status.

Further analysis: formal testing of region-by-satiety and region-by-drug interactions

Of course, the presence of qualitatively different responses in the three regions is not directly indicative that the regions show significantly different profiles of activation. We therefore extracted average data from the entire spheres of interest (originally identified using high-calorie vs low-calorie contrast, as above) and tested these regional interactions post hoc by entering caloriespecific activations (that is, the magnitude of difference in response to high-calorie, compared to low-calorie, food images) into a $3 \times 2 \times 2$ ANOVA. This is not independent of the voxelwise analyses described above but serves as a complementary analysis aimed at establishing the significance of regional differences already described. The ANOVA embodied three factors: region (three levels: amygdala, hypothalamus, and striatum), satiety (two levels: fasted and fed) and drug treatment (two levels: placebo and sibutramine), and also included a Greenhouse-Geisser correction. The ANOVA showed significant region-by-drug $\left(F_{(2,46)}=6.5 ; p\right.$ (one-tailed $\left.)=0.005\right)$ and region-by-satiety $\left(F_{(2,46)}=3 ; p(\right.$ one-tailed $\left.)=0.04\right)$ interactions. Post hoc tests confirmed significant region-by-satiety $\left(F_{(1,23)}=11.5\right.$; $p$ (onetailed $)=0.004)$ and region-by-drug $\left(F_{(1,23)}=3.9 ; p\right.$ (onetailed $)=0.03$ ) interactions when comparing hypothalamus and ventral striatum as well as significant region-by-drug $\left(F_{(1,23)}=\right.$ $10 ; p($ one-tailed $)=0.008$ ) and near-significant region-by-satiety $\left(F_{(1,23)}=2 ; p(\right.$ one-tailed $\left.)=0.085\right)$ interactions when comparing amygdala with ventral striatum.

Thus, the results show a significant double dissociation across hypothalamic and ventral striatal responses and a strong trend toward double dissociation between amygdala and ventral striatum. Hypothalamic and amygdala responses did not significantly differ.

\section{Discussion}

In summary, we have shown that the modulatory impact of sibutramine and satiety differs significantly across hypothalamus, amygdala, and ventral striatum. Sibutramine, as well as producing the expected reductions in weight and food consumption, was associated with an attenuation of calorie-specific responding 
in the hypothalamus and amygdala, regardless of satiety. Moreover, the magnitude of hypothalamic modulation correlated with subsequent ad libitum consumption and weight change. Striatal responses, which were sensitive to the effects of satiety, but not drug, also correlated with subsequent ad libitum consumption. This double dissociation suggests functionally distinct roles of these regions responding to food-related stimuli.

The pattern of brain responses to images of high-calorie foods, compared to low-calorie, bland foods, is consistent with previous observations (Killgore et al., 2003; Schur et al., 2009). While other regions, including prefrontal cortex, thalamus, and cerebellum (Killgore et al., 2003) and hippocampus (Pelchat et al., 2004), have also shown such sensitivity, we initially focused upon activations in midbrain, ventral striatum, amygdala, hypothalamus, and insula, which have been consistently shown to be responsive to high-calorie food images and were found to be so in the current study. We should acknowledge that the imaging parameters used did not optimize acquisition of data from orbitofrontal cortex, which previous work has implicated in such tasks (Killgore and Yurgelun-Todd, 2005; Porubská et al., 2006; Rothemund et al., 2007; Führer et al., 2008; Schienle et al., 2009). The implications of the distinct regional effects of drug and satiety are considered below.

\section{Ventral striatum}

The impact of fasting on neuronal responses to food stimuli has been studied using functional MRI (e.g., Goldstone et al., 2009), and while the precise brain regions showing such an effect have varied across studies, the general pattern is one of an eating-induced reduction in activation. This is to be expected for regions where activation signals food's value or desirability, which would diminish with satiety. It fits with the observations made here in the ventral striatum, which was sensitive to participants' current state of hunger. However, in contrast to amygdala and hypothalamus, its activity was not modified over the 2 week period of sibutramine treatment. Ventral striatal responses have been implicated in reward processing (O'Doherty, 2004), specifically showing responses to stimuli that predict imminent reward. It has been suggested that this region codes the motivating properties of stimuli (Berridge and Kringelbach, 2008; Berridge, 2009), a suggestion consistent with the eating-induced reduction in response observed here. We have previously shown that more rewarding food pictures are associated with greater striatal responses in leptin-deficient individuals (who show extreme motivation toward food stimuli) and that this relationship is stronger in the fasted state, being attenuated by eating but only following leptinreplacement treatment (Farooqi et al., 2007). Intriguingly, the impact of leptin on ventral striatal responses may derive, in part, from its modulation of dopamine release (Krügel et al., 2003).

\section{Hypothalamus}

The hypothalamic calorie-specific response was insensitive to the participants' current state of satiety but was attenuated by drug treatment. This occurred in the context of the drug's expected therapeutic effects on body weight, fat mass, and eating behavior. These anthropometric and behavioral effects of sibutramine were related here directly to its effects on brain activation. Individuals who experienced greater reductions of body weight and ad libitum food intake showed greater drug-related suppression of calorie-specific hypothalamic responses. These data strongly suggest that changes in eating behavior and body weight attributable to sibutramine treatment are mediated or caused by changes in functional responding in the hypothalamus.

It appears that the impact of the drug on hypothalamic responding may be key to understanding its beneficial effect in the treatment of obesity, especially given the correlation between the hypothalamic effects of sibutramine and its clinical efficacy. The finding complements recent work suggesting that, in the context of manipulation of PYY, greater levels of hypothalamic activity predict subsequent eating behavior (Batterham et al., 2007). This is in keeping with experimental studies in rodents, which show that central pathways involving the hypothalamus are critical to the regulation of appetite and weight (Morton et al., 2006), and that serotonergic effects on food intake are mediated predominantly via pro-opiomelanocortin (POMC)-expressing neurons in the arcuate nucleus of the hypothalamus (Zhou et al., 2005; Heisler et al., 2006). It is compatible too with observations 
a
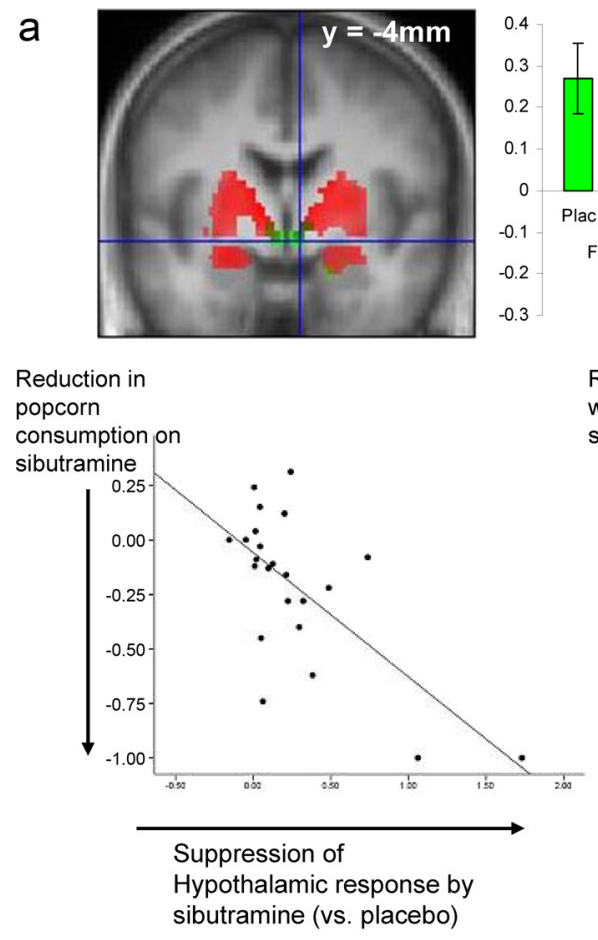

Reduction in

weight on

sibtramine

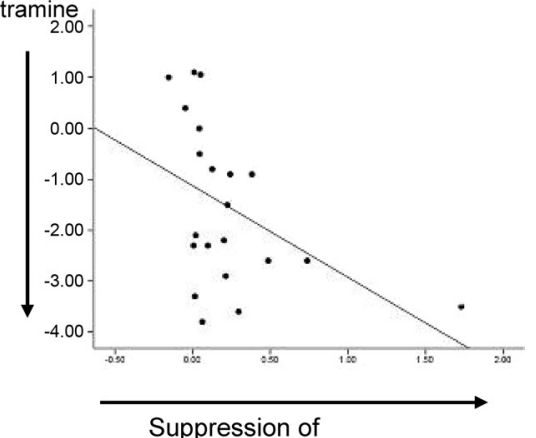

Suppression of

Hypothalamic response by sibutramine (vs. placebo) b

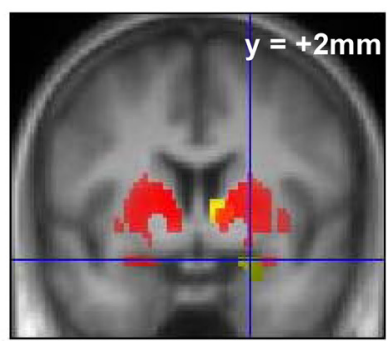

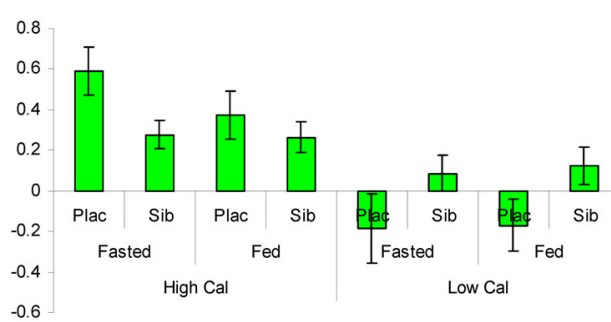

$-0.6$

nucleus of the hypothalamus. While the spatial resolution of fMRI does not allow us to distinguish between these neuronal groups, it is intriguing to observe that the overall response in our study was one of suppression. However, this observation should be qualified by adding that while the overall drug-induced response is one of suppression, it remains feasible that the impact of sibutramine is to elevate baseline or tonic hypothalamic activity, leading both to an increase in satiety and to an attenuated response to the food-related stimuli that would normally induce a desire to eat. We can only speculate on this, but the clear impact of the drug on the hypothalamus and the relationship of this impact to weight and food-related behaviors are strongly suggestive that the neural basis of sibutramine's efficacy is, at least, in part consequent upon its ability to modulate hypothalamic responses.

Our combined imaging and behavioral findings are compatible with a causal relationship whereby reduced brain activation leads to reduced food intake and body weight. Of course, it would be beneficial ultimately if imaging observations were predictive of clinical efficacy. With this in mind, we examined specifically the group of participants that received placebo first. Individuals with greater hypothalamic activation on placebo were found to be more sensitive to subsequent sibutramine treatment in terms of its efficacy in producing weight loss.

Figure 4. $\quad \boldsymbol{a}$, Hypothalamic responses to experimental manipulations (image type and sibutramine status). Top left, A corona section of the group average structural $\mathrm{MRI}$ at $4 \mathrm{~mm}$ posterior to the anterior commissure. Superimposed in red are the local regions showing a calorie-specific (high-calorie vs low-calorie images) effect ( $p<0.05$, corrected), and in green ( $p<0.01$, uncorrected) are the hypothalamic regions in which this calorie-specific response is significantly attenuated by sibutramine. Top right, The plots show estimates of stimulus-related brain activation for each of the eight key conditions, divided by image type (high calorie/low calorie), satiety state (fasted/fed), and treatment (sibutramine/placebo). The $y$-axes show the effect sizes in the ventral striatal region shown or parameter estimates derived from the general linear model (units are arbitrary). As can be seen there is a strong effect of image type and of sibutramine but not of fasted/fed state. Bottom, Scatter plots showing the associations between the effect of sibutramine on suppression of hypothalamic activation by high-calorie food images ( $x$-axis) and its effects on the proportion of ad libitum popcorn consumed on sibutramine compared to placebo (left) and on body weight in kilograms (right). In both graphs, a value of greater than zero on the $x$-axis indicates a suppression of activation on sibutramine compared to placebo; a value of less than zero on the $y$-axis indicates that there was a greater reduction in snacking or weight for sibutramine compared with for placebo. The graphs show that participants who demonstrated greater suppression of hypothalamic activation by sibutramine also showed greater treatment-related reduction in popcorn snacking and body weight. $\boldsymbol{b}$, Amygdala responses to experimental manipulations (image type and sibutramine status). Left, A coronal section of the group average structural MRI at $2 \mathrm{~mm}$ anterior to the anterior commissure. Superimposed in red are the local regions showing a calorie-specific (high-calorie vs low-calorie images) response ( $p<0.05$, corrected), and in green (at crosshairs) is the amygdala region in which this calorie-specific response is significantly attenuated by sibutramine ( $p<0.01$, uncorrected). Right, The plots show estimates of stimulus-related brain activation for each of the eight key conditions, divided by image type (high calorie/low calorie), satiety state (fasted/fed), and treatment (sibutramine/placebo). The $y$-axis show the effect sizes in the ventral striatal region shown or parameter estimates derived from the general linear model (units are arbitrary). As can be seen, there is a strong effect of image type and of sibutramine but not of fasted/fed state.

of hypothalamic serotonin release following systemic sibutramine administration (Gundlah et al., 1997). It is noteworthy that observations in mice (Heisler et al., 2006) and the emerging models of serotonergic impact on hypothalamic neurons (Garfield and Heisler, 2009) suggest that upregulation of serotonin would have both an excitatory effect on POMC cells and an inhibitory effect on Agouti-related protein neurons, both within the arcuate

\section{Amygdala}

The pattern of response in amygdala, a region that has repeatedly shown sensitivity to food-related stimuli (Killgore et al., 2003; Holsen et al., 2005, 2006; Stoeckel et al., 2008; Schur et al., 2009) was comparable to that found in the hypothalamus. Previous observations of amygdala responses to food-related stimuli, suggest that its activation is related to the value placed on foods by individuals. This is shown by elevated responses in obese people (Stoeckel et al., 2008) and its unusually persistent activity following a meal in individuals with Prader-Willi syndrome (Holsen et al., 2006). Furthermore, an infusion of ghrelin, which heightens appetite, increases the amygdala response to food images (Malik et al., 2008). More generally, amygdala activation correlates well with the rewardpredicting value assigned to a stimulus (Gottfried et al., 2003), and evidence from animals shows clearly that damage to amygdala prevents the attenuation of reward-acquiring behaviors when the reward itself is no longer valued, for example, as a consequence of overfeeding (Johnson et al., 2009). Our findings too are compatible with the amygdala encoding the value that 
participants attach to the images representing foods. In particular, the effect of sibutramine is noteworthy, given that the amygdala has abundant serotonergic input and receptors (Hariri et al., 2006) and enhancing serotonin neurotransmission may attenuate amygdala activity through potentiation of GABAergic inhibition (Rainnie, 1999).

It has previously been shown that the amygdala and hypothalamus are less responsive to food pictures when participants lose weight (Rosenbaum et al., 2008). We considered the possibility that the reductions in activation reported here reflects a nonspecific effect of weight loss. However, in a post hoc analysis of those participants who received sibutramine first, while weight reduction was maintained throughout the second period of treatment with placebo, the treatment effect on hypothalamic and amygdala activation remained significant. This result indicates that reduced body weight alone did not attenuate brain responses to food stimuli in the placebo condition.

The problems posed by obesity have driven researchers to consider its origins not solely in terms of basic homeostatic drives invoking hunger and satiety but also in terms of the higher-level cognitive processes such as learning, wanting, and liking that might arise from and shape these drives. In this regard, functional neuroimaging has been playing an increasing role in how we investigate obesity and its causes. In the future, it is likely also to contribute to the development and testing of new treatments. The current study elucidates the neural modulations produced by two independent experimental manipulations of participants' drive to eat: satiety and treatment with an anti-obesity drug. Across three key regions, we observed dissociable patterns of response, which were predictive of subsequent food consumption. These data suggest fundamentally different contributions of these regions to eating behavior. Our results also point to the possibility of developing behavioral and brain functional markers or predictors of therapeutic response that might be of clinical value in identifying individual patients most likely to benefit from personalized or stratified treatment with centrally acting anti-obesity drugs.

\section{References}

Batterham RL, ffytche DH, Rosenthal JM, Zelaya FO, Barker GJ, Withers DJ, Williams SC (2007) PYY modulation of cortical and hypothalamic brain areas predicts feeding behaviour in humans. Nature 450:106-109.

Beaver JD, Lawrence AD, van Ditzhuijzen J, Davis MH, Woods A, Calder AJ (2006) Individual differences in reward drive predict neural responses to images of food. J Neurosci 26:5160-5166.

Berridge KC (2009) 'Liking' and 'wanting' food rewards: brain substrates and roles in eating disorders. Physiol Behav 97:537-550.

Berridge KC, Kringelbach ML (2008) Affective neuroscience of pleasure: reward in humans and animals. Psychopharmacology 199:457-480.

Coletta M, Platek S, Mohamed FB, van Steenburgh JJ, Green D, Lowe MR (2009) Brain activation in restrained and unrestrained eaters: an fMRI study. J Abnorm Psychol 118:598-609.

Cornier MA, Von Kaenel SS, Bessesen DH, Tregellas JR (2007) Effects of overfeeding on the neuronal response to visual food cues. Am J Clin Nutr 86:965-971.

Cornier MA, Salzberg AK, Endly DC, Bessesen DH, Rojas DC, Tregellas JR (2009) The effects of overfeeding on the neuronal response to visual food cues in thin and reduced-obese individuals. PLoS One 4:e6310.

Farooqi IS, Bullmore E, Keogh J, Gillard J, O'Rahilly S, Fletcher PC (2007) Leptin regulates striatal regions and human eating behavior. Science 317:1355.

Führer D, Zysset S, Stumvoll M (2008) Brain activity in hunger and satiety: an exploratory visually stimulated FMRI study. Obesity 16:945-950.

Garfield AS, Heisler LK (2009) Pharmacological targeting of the serotonergic system for the treatment of obesity. J Physiol 587:49-60.

Goldstone AP, de Hernandez CG, Beaver JD, Muhammed K, Croese C, Bell G, Durighel G, Hughes E, Waldman AD, Frost G, Bell JD (2009) Fasting biases brain reward systems towards high-calorie foods. Eur J Neurosci 30:1625-1635.

Gottfried JA, O’Doherty J, Dolan RJ (2003) Encoding predictive reward value in human amygdala and orbitofrontal cortex. Science 301:1104-1107.

Gundlah C, Martin KF, Heal DJ, Auerbach SB (1997) In vivo criteria to differentiate monoamine reuptake inhibitors from releasing agents: sibutramine is a reuptake inhibitor. J Pharmacol Exp Ther 283:581-591.

Hansen D, Astrup A, Toubro S, Finer N, Kopelman P, Hilsted J, Rössner S, Saris W, Van Gaal L, James W, Goulder M, for the STORM Study Group (2001) Predictors of weight loss and maintenance during 2 years of treatment by sibutramine in obesity. Results from the European multi-centre STORM trial. Sibutramine Trial of Obesity Reduction and Maintenance. Int J Obes Relat Metab Disord 25:496-501.

Hariri AR, Drabant EM, Weinberger DR (2006) Imaging genetics: perspectives from studies of genetically driven variation in serotonin function and corticolimbic affective processing. Biol Psychiatry 59:888-897.

Heisler LK, Jobst EE, Sutton GM, Zhou L, Borok E, Thornton-Jones Z, Liu HY, Zigman JM, Balthasar N, Kishi T, Lee CE, Aschkenasi CJ, Zhang CY, Yu J, Boss O, Mountjoy KG, Clifton PG, Lowell BB, Friedman JM, Horvath T, et al. (2006) Serotonin reciprocally regulates melanocortin neurons to modulate food intake. Neuron 51:239-249.

Holsen LM, Zarcone JR, Thompson TI, Brooks WM, Anderson MF, Ahluwalia JS, Nollen NL, Savage CR (2005) Neural mechanisms underlying food motivation in children and adolescents. Neuroimage 27:669-676.

Holsen LM, Zarcone JR, Brooks WM, Butler MG, Thompson TI, Ahluwalia JS, Nollen NL, Savage CR (2006) Neural mechanisms underlying hyperphagia in Prader-Willi syndrome. Obesity 14:1028-1037.

Johnson AW, Gallagher M, Holland PC (2009) The basolateral amygdala is critical to the expression of Pavlovian and instrumental outcome-specific reinforcer devaluation effects. J Neurosci 29:696-704.

Killgore WD, Yurgelun-Todd DA (2005) Body mass predicts orbitofrontal activity during visual presentations of high-calorie foods. Neuroreport 16:859-863.

Killgore WD, Yurgelun-Todd DA (2007) Positive affect modulates activity in the visual cortex to images of high calorie foods. Int J Neurosci 117:643-653.

Killgore WD, Yurgelun-Todd DA (2010) Sex differences in cerebral responses to images of high versus low-calorie food. Neuroreport 21:354-358.

Killgore WD, Young AD, Femia LA, Bogorodzki P, Rogowska J, YurgelunTodd DA (2003) Cortical and limbic activation during viewing of highversus low-calorie foods. Neuroimage 19:1381-1394.

Krügel U, Schraft T, Kittner H, Kiess W, Illes P (2003) Basal and feedingevoked dopamine release in the rat nucleus accumbens is depressed by leptin. Eur J Pharmacol 482:185-187.

LaBar KS, Gitelman DR, Parrish TB, Kim YH, Nobre AC, Mesulam MM (2001) Hunger selectively modulates corticolimbic activation to food stimuli in humans. Behav Neurosci 115:493-500.

Lean ME (2001) How does sibutramine work? Int J Obes Relat Metab Disord 25:S8-S11.

Maldjian JA, Laurienti PJ, Kraft RA, Burdette JH (2003) An automated method for neuroanatomic and cytoarchitectonic atlas-based interrogation of fMRI data sets. Neuroimage 19:1233-1239.

Malik S, McGlone F, Bedrossian D, Dagher A (2008) Ghrelin modulates brain activity in areas that control appetitive behavior. Cell Metab 7:400-409.

McCaffery JM, Haley AP, Sweet LH, Phelan S, Raynor HA, Del Parigi A, Cohen R, Wing RR (2009) Differential functional magnetic resonance imaging response to food pictures in successful weight-loss maintainers relative to normal-weight and obese controls. Am J Clin Nutr 90:928-934.

Morton GJ, Cummings DE, Baskin DG, Barsh GS, Schwartz MW (2006) Central nervous system control of food intake and body weight. Nature 443:289-295.

Murray GK, Corlett PR, Clark L, Pessiglione M, Blackwell AD, Honey G, Jones PB, Bullmore ET, Robbins TW, Fletcher PC (2008) Substantia nigra/ventral tegmental reward prediction error disruption in psychosis. Mol Psychiatry 13:239, 267-276.

Nisoli E, Carruba MO (2000) An assessment of the safety and efficacy of sibutramine, an anti-obesity drug with a novel mechanism of action. Obes Rev 1:127-139.

O’Doherty JP (2004) Reward representations and reward-related learning 
in the human brain: insights from neuroimaging. Curr Opin Neurobiol 14:769-776.

Pelchat ML, Johnson A, Chan R, Valdez J, Ragland JD (2004) Images of desire: food-craving activation during fMRI. Neuroimage 23:14861493.

Piech RM, Lewis J, Parkinson CH, Owen AM, Roberts AC, Downing PE, Parkinson JA (2009) Neural correlates of appetite and hunger-related evaluative judgments. PLoS One 4:e6581.

Porubská K, Veit R, Preissl H, Fritsche A, Birbaumer N (2006) Subjective feeling of appetite modulates brain activity: an fMRI study. Neuroimage 32:1273-1280.

Rainnie DG (1999) Serotonergic modulation of neurotransmission in the rat basolateral amygdala. J Neurophysiol 82:69-85.

Rosenbaum M, Sy M, Pavlovich K, Leibel RL, Hirsch J (2008) Leptin reverses weight loss-induced changes in regional neural activity responses to visual food stimuli. J Clin Invest 118:2583-2591.

Rothemund Y, Preuschhof C, Bohner G, Bauknecht HC, Klingebiel R, Flor H, Klapp BF (2007) Differential activation of the dorsal striatum by highcalorie visual food stimuli in obese individuals. Neuroimage 37:410-421.

Schienle A, Schäfer A, Hermann A, Vaitl D (2009) Binge-eating disorder: reward sensitivity and brain activation to images of food. Biol Psychiatry 65:654-661.

Schur EA, Kleinhans NM, Goldberg J, Buchwald D, Schwartz MW, Maravilla K (2009) Activation in brain energy regulation and reward centers by food cues varies with choice of visual stimulus. Int J Obes (Lond) 33:653-661.

Smeets PA, de Graaf C, Stafleu A, van Osch MJ, Nievelstein RA, van der Grond J (2006) Effect of satiety on brain activation during chocolate tasting in men and women. Am J Clin Nutr 83:1297-1305.

Stice E, Spoor S, Bohon C, Small DM (2008) Relation between obesity and blunted striatal response to food is moderated by TaqIA Al allele. Science 322:449-452.

Stoeckel LE, Weller RE, Cook EW 3rd, Twieg DB, Knowlton RC, Cox JE (2008) Widespread reward-system activation in obese women in response to pictures of high-calorie foods. Neuroimage 41:636-647.

Uher R, Treasure J, Heining M, Brammer MJ, Campbell IC (2006) Cerebral processing of food-related stimuli: effects of fasting and gender. Behav Brain Res 169:111-119.

Zhou L, Williams T, Lachey JL, Kishi T, Cowley MA, Heisler LK (2005) Serotonergic pathways converge upon central melanocortin systems to regulate energy balance. Peptides 26:1728-1732. 